\title{
Hole dynamics in a quantum-well state at $\mathrm{Na} / \mathrm{Cu}(111)$
}

\author{
E. V. Chulkov, ${ }^{1,2}$ J. Kliewer, ${ }^{3}$ R. Berndt,${ }^{3}$ V. M. Silkin, ${ }^{1}$ B. Hellsing, ${ }^{4}$ S. Crampin, ${ }^{5}$ and P. M. Echenique ${ }^{1,2}$ \\ ${ }^{1}$ Donostia International Physics Center (DIPC), Paseo de Manuel Lardizabal, 4, 20018 San Sebastián/Donostia, Basque Country, Spain \\ ${ }^{2}$ Departamento de Física de Materiales and Centro Mixto CSIC-UPV/EHU, Facultad de Ciencias Químicas, UPV/EHU, \\ Apartado Postal 1072, 20080 San Sebastián/Donostia, Basque Country, Spain \\ ${ }^{3}$ Institut für Experimentelle und Angewandte Physik, Christian-Albrechts-Universität zu Kiel, D-24098 Kiel, Germany \\ ${ }^{4}$ Experimental Physics, Chalmers University of Technology and Göteborg University, S-412 96 Göteborg, Sweden \\ ${ }^{5}$ Department of Physics, University of Bath, Bath BA2 7AY, United Kingdom
}

(Received 15 April 2003; published 26 November 2003)

\begin{abstract}
Theoretical calculations and scanning-tunneling spectroscopy measurements of the hole lifetime broadening, $\tau^{-1}$, in a quantum-well state for 0.95 and 1.0 monolayers of $\mathrm{Na}$ on $\mathrm{Cu}(111)$ are reported. A model potential is proposed for calculating quantum-well states in a monolayer on metal surfaces. The inelastic electron-electron contribution, $\tau_{e-e}^{-1}$, is evaluated within the $G W$ approximation by using eigenfunctions and eigenenergies obtained with this model potential. The electron-phonon contribution, $\tau_{e \text {-ph }}^{-1}$, is computed by employing Debye and Einstein models as well as a first-principle ultrasoft pseudopotential method. The obtained theoretical results are in excellent agreement with experimental data, both showing a surprisingly large difference in the lifetime broadening for 0.95 and 1.0 monolayers which is attributed mostly to changes in the electronic structure.
\end{abstract}

DOI: 10.1103/PhysRevB.68.195422

PACS number(s): 73.20.At, 71.45.Gm, 73.50.Gr, 78.47.+p

\section{INTRODUCTION}

The electronic properties of ultrathin metal films adsorbed on metal surfaces are of fundamental interest due to quantum size effects. One aspect of these occurs in films with thicknesses ranging from a fraction of a saturated atomic monolayer to several monolayers, which may be prepared via evaporation onto a supporting substrate. If the substrate has a local band gap in the direction normal to the surface, it is possible for electrons to be trapped in quantum-well states (QWS). These QWS, which form two-dimensional (2D) electron gases with metal densities, have been observed and investigated by angle-resolved photoemission spectroscopy (PES), ${ }^{1-3}$ two-photon photoemission technique (2PPE), ${ }^{4}$ and by scanning tunneling microscopy (STM). ${ }^{5}$ In recent years intensified experimental and theoretical studies of quantumwell states have been performed for ultrathin films [around 1 monolayer (ML)] of sodium atoms on the $\mathrm{Cu}(111)$ surface. ${ }^{1,5-9}$ Intriguingly, the binding energies of the QWS are strongly affected by the Na coverage. ${ }^{7}$ For example for the coverages of interest below, 1 and 0.95 monolayers, binding energies of $-0.127 \mathrm{eV}$ and $-0.042 \mathrm{eV}$ with respect to the Fermi level were observed with scanning tunneling spectroscopy ${ }^{5}$ confirming previous data from photoelectron spectroscopy ${ }^{7}$ which yielded values of approximately $-100 \mathrm{meV}$ and $-75 \mathrm{meV}$. The QWS linewidths have first been measured by PES (Ref. 7) and interpreted in terms of inelastic electron-phonon $\tau_{e-\text { ph }}^{-1}$ and elastic electron-defect (impurity) scattering. ${ }^{7,8}$ The inelastic electron-electron contribution $\tau_{e-e}^{-1}$ was estimated within the 3D free-electron-gas mode $^{10}$ and was ignored as negligibly small. The measured, rather abrupt increase of the linewidth of about $16 \mathrm{meV}$ when increasing the coverage from $0.95 \mathrm{ML}$ to $1 \mathrm{ML}$ was attributed to inhomogeneous broadening (impurity or defect scattering). ${ }^{7}$

In this paper we provide two contributions. First, we pro- pose a model potential which gives a realistic band structure of $\mathrm{Na} / \mathrm{Cu}(111)$, and calculate the inelastic electron-electron contribution for $0.95 \mathrm{ML}$ and $1.0 \mathrm{ML}$ of $\mathrm{Na}$ on $\mathrm{Cu}(111) .{ }^{11}$ We show that this contribution cannot be neglected; that, in fact, it accounts for the measured difference in the lifetime broadening that exists between these two coverages. We also evaluate the electron-phonon contribution by using both Debye and Einstein models as well as a first-principle method combined with the Einstein model.

Second, we perform scanning tunneling spectroscopy of 0.95- and 1.0-ML $\mathrm{Na}$ on $\mathrm{Cu}(111)$ extracting the QWS binding energies and the hole lifetimes. The lifetime of the hole in the $\bar{\Gamma}$ point was obtained from a line shape analysis and compared with theoretical estimates. Until recently, the lifetimes of holes in electronic states localized on metal surfaces have been determined exclusively using photoelectron spectroscopy, as reviewed by Matzdorf. ${ }^{12}$ More recently, scanning tunneling spectroscopy measurements have also addressed hole dynamics and the lifetimes of surface state holes on noble metal surfaces have been determined. ${ }^{13,14}$ An important asset of the STM in this context is the ability to verify surface perfection before taking spectroscopic data, allowing undesirable effects due to defect scattering that have affected PES results to be avoided. Indeed recent PES experiments conducted specifically to minimize measurement times and thus surface contamination and damage have confirmed the STM data. ${ }^{15}$ While the original STM approach was limited to specific points of the band structure, the technique has also been extended to probe various energies by making use of artificial atomic arrays on different metal surfaces, ${ }^{16-18}$ and via an alternative approach first indicated by Avouris et al. ${ }^{19}$ that uses the spatial decay of electron interference patterns observed in STM images to measure both electron and hole lifetimes. ${ }^{20-22}$

The paper is organized as follows. In Sec. II the model potential is presented and the most important features of the 
calculations of $\tau_{e-e}^{-1}$ based on the self-energy formalism of many-body theory are briefly outlined. In this section we also give a brief discussion of different methods of the evaluation of $\tau_{e \text {-ph }}^{-1}$ and report the calculation results. Experimental procedures and measured characteristics are presented in Sec. III. In Sec. IV we discuss the theoretical and experimental results, and in Sec. V the conclusions of the work are given.

\section{THEORETICAL PART}

\section{A. Model potential}

A model potential that for clean metal surfaces reproduces exactly the energy gap and binding energy of both the $s$ $-p_{z}$ surface state and the first image-potential state has recently been proposed in Refs. 23 and 24 . This potential varies in the $z$ direction perpendicular to the surface and is uniform in the $(x, y)$ plane parallel to the surface, and has been used successfully in calculations of image (surface) state electron (hole) lifetimes on clean metal surfaces ${ }^{14,25,26}$ and also metal surfaces covered with a single alkali adatom. ${ }^{27}$ Here we extend this model potential to systems which consist of an atomic monolayer adsorbed on a metal surface and construct the potential for 0.95 and 1.0 monolayers of $\mathrm{Na}$ on $\mathrm{Cu}(111)$. The potential also varies only in the $z$ direction and has the form

$$
\begin{gathered}
V_{1}(z)=A_{10}+A_{1} \cos \left(\frac{2 \pi}{a_{s}} z\right), z<D, \\
V_{2}(z)=A_{20}+A_{2} \cos [\beta(z-D)], D<z<z_{s}, \\
V_{i n t}(z)=A_{i n t}+B_{i n t} z, z_{s}<z<z_{a d}, \\
V_{a d}(z)=A_{a d 0}+A_{a d} \cos \left[\beta_{a d}\left(z-D_{a d}\right)\right], z_{a d}<z<z_{1}, \\
V_{3}(z)=A_{3} \exp \left[-\alpha\left(z-z_{1}\right)\right], z_{1}<z<z_{i m}, \\
V_{4}(z)=\frac{\exp \left[-\lambda\left(z-z_{i m}\right)\right]-1}{4\left(z-z_{i m}\right)}, z_{i m}<z .
\end{gathered}
$$

Here $a_{s}$ is the interlayer spacing, $D$ and $D_{a d}$ denote the position of the surface atomic layer of the substrate and of the atomic adlayer position, respectively. Equation (1) describes the potential in bulk $\mathrm{Cu}$ normal to the (111) surface, and is terminated at the surface atomic layer of the substrate. The parameters $A_{1}$ and $A_{10}$ of this equation reproduce the width and position of the energy gap of the substrate. $V_{2}(z)$ and $V_{\text {int }}(z)$ represent the potential in the substrate-adlayer interface region, while Eq. (4) describes the potential at the adlayer. The adlayer-vacuum interface region is represented by $V_{3}(z)$, and finally $V_{4}(z)$ is the image potential.

Equations (1)-(6) have 17 parameters: $A_{1}, A_{10}, A_{2}, A_{20}$, $\beta, z_{s}, A_{\text {int }}, B_{i n t}, z_{a d}, A_{a d}, A_{a d 0}, \beta_{a d}, A_{3}, \alpha, z_{1}, \lambda$, and $z_{i m}$; only six of them are independent. We choose the six parameters $A_{1}, A_{10}, A_{2}, \beta, z_{s}$, and $\beta_{a d}$ as adjustable ones, the others being determined from the requirement of continuity of the potential and its first derivative everywhere in space. The four first parameters $A_{1}, A_{10}, A_{2}$, and $\beta$ taken

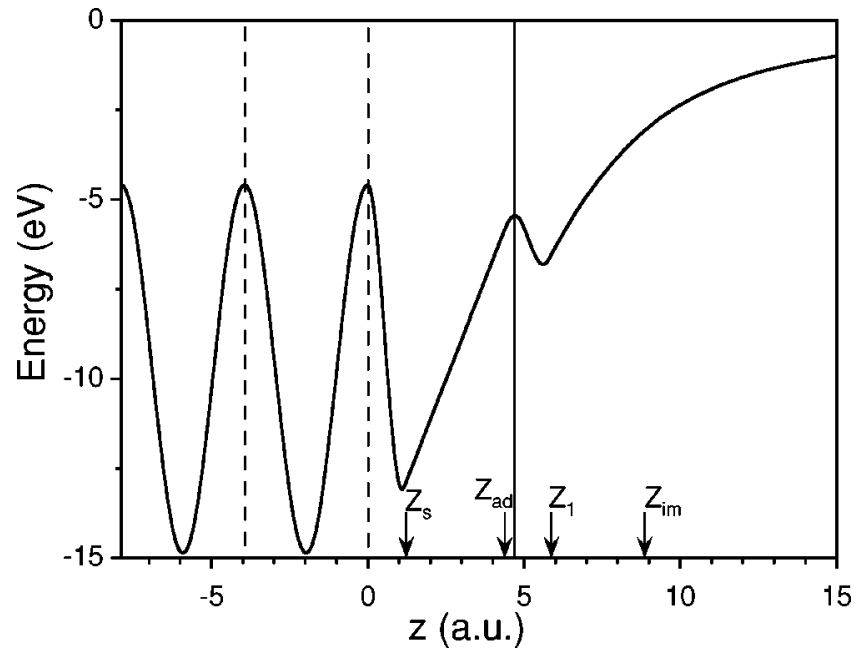

FIG. 1. Model potential for $1 \mathrm{ML}$ of $\mathrm{Na}$ on $\mathrm{Cu}(111)$ along the surface normal. Vertical lines represent atomic layer positions: solid line indicates the $\mathrm{Na}$ overlayer position and dashed lines show the copper atomic layer positions. For $z_{s}, z_{a d}, z_{1}$, and $z_{i m}$ see the text.

from the potential fitted to low-temperature measured data for clean $\mathrm{Cu}(111)$ (Refs. 14 and 24) are not adjustable parameters for the $\mathrm{Na} / \mathrm{Cu}(111)$ system; only $z_{s}$ and $\beta_{a d}$ are adjustable. They are determined from a fit of the calculated binding energy of the QWS, $E_{0}$, and the first image state, $E_{1}$, to values $E_{0}$ and $E_{1}$ obtained from STM (Ref. 5) and 2PPE (Ref. 6) measurements, respectively. However, to achieve perfect fitting of $E_{0}$ and $E_{1}$ we also vary slightly the parameter $z_{a d}$ first fixed at $z_{a d}=-\pi /\left(4 \beta_{a d}\right)$ relative to the adlayer position. The fitted intermediate point $z_{s}$ is slightly smaller than $z_{1}=5 \pi /(4 \beta)$ fixed for the clean surface $\mathrm{Cu}(111) .{ }^{28}$ In our model the image plane position $z_{i m}$ is not a fitting parameter, it is obtained from Eqs. (1)-(6) as

$$
z_{i m}=-\frac{\ln \left[-\lambda /\left(4 A_{3}\right)\right]}{\alpha}+z_{1}
$$

where the intermediate point $z_{1}$ determined relative to the adlayer position is fixed at $z_{1}=5 \pi /\left(4 \beta_{a d}\right)$.

In Fig. 1 we show the model potential calculated for $1-\mathrm{ML} \mathrm{Na} / \mathrm{Cu}(111)$; the potential evaluated for $0.95-\mathrm{ML} \mathrm{Na} /$ $\mathrm{Cu}(111)$ has a similar form. The $A_{a d}$ amplitude $\left[A_{a d}\right.$ $=0.68 \mathrm{eV}$ for $1-\mathrm{ML} \mathrm{Na} / \mathrm{Cu}(111)]$ is much smaller than that for $\mathrm{Cu}$ and is close to the potential amplitude for clean $\mathrm{Na}(110)\left[A_{1}^{\mathrm{Na}}=0.67 \mathrm{eV}\right.$ (Ref. 24)]. It is interesting to note that the potential of Fig. 1 looks quite similar to the local part of the self-consistent screened pseudopotential obtained for $\mathrm{Al}(001)+c(2 \times 2) \mathrm{Na}^{29}$ The distinction between these two potentials results mostly from the different energy position of the potential at the Na overlayer with respect to the potential position in the substrate region. In Table I we show the computed and experimental results for the quantum well and image potential states for $1-\mathrm{ML} \mathrm{Na} / \mathrm{Cu}(111)$ and the QWS energy for $0.95 \mathrm{ML} \mathrm{Na} / \mathrm{Cu}(111)$. We also give the evaluated image plane position, $z_{i m}$, which is important for the correct description of binding energies of image states. ${ }^{30}$ We do not display image potential states energies and $z_{i m}$ evaluated for 
TABLE I. Theoretical and experimental energies (in eV) of the quantum well, $E_{0}$, and the image potential states for the Na overlayers on $\mathrm{Cu}(111) . E_{0}$ is given with respect to the Fermi level and $E_{1}, E_{2}, E_{3}$ with respect to the vacuum level. The obtained image plane position, $z_{i m}$ (in a.u.), is given with respect to the Na overlayer position

\begin{tabular}{lccc}
\hline \hline Overlayer (ML) & & Theory & Experiment \\
\hline 1 & $E_{0}$ & -0.127 & -0.127 (Ref. 5), \\
& & & $-0.11 \pm 0.03$ (Ref. 6), \\
& & & -0.10 (Ref. 7) \\
& $E_{1}$ & -0.72 & $-0.72 \pm 0.03$ (Ref. 6) \\
& $E_{2}$ & -0.22 & $-0.27 \pm 0.03$ (Ref. 6) \\
& $E_{3}$ & -0.10 & $-0.13 \pm 0.04$ (Ref. 6) \\
& $z_{\text {im }}$ & 3.67 & \\
\hline \hline & $E_{0}$ & -0.042 & -0.042 (Ref. 5) \\
\hline
\end{tabular}

$0.95 \mathrm{ML} \mathrm{Na} / \mathrm{Cu}(111)$ since they are similar to those for $1-\mathrm{ML}$ $\mathrm{Na} / \mathrm{Cu}(111)$. With this $z_{\text {im }}$ we obtain image state energies $E_{2}$ and $E_{3}$ in good agreement with 2PPE results. ${ }^{6,31}$

For the description of the quantum-well state for 1-ML $\mathrm{Na} / \mathrm{Cu}(111)$ Lindgren and Walldén proposed a onedimensional potential ${ }^{1}$ which coincides with ours in the $\mathrm{Cu}$ bulk region and is constant at the $\mathrm{Na}$ adlayer position and in both the substrate-adlayer and adlayer-vacuum interface regions. They reproduced the experimental binding energy of the QWS, but, as was shown by Fischer et al., ${ }^{6,31}$ their potential does not simultaneously describe the binding energies of the QWS and the image states. Furthermore, Fischer et al. found that no set of the parameters in Lindgren and Walldén's potential exists that simultaneously describes the measured binding energies $E_{0}, E_{1}, E_{2}$, and $E_{3}$ for 1-ML Na/ $\mathrm{Cu}(111)$. The authors of Refs. 6 and 31 attributed these results to the potential used in Ref. 1 being oversimplified.

\section{B. Electron-electron contribution}

Within a many-body theory the inelastic electron-electron contribution, $\tau_{e-e}^{-1}\left(\Gamma_{e-e}\right)$, to the lifetime broadening (linewidth) of a hole $\left(E_{0}<E_{F}\right)$ in a quantum state $\psi_{0}(\mathbf{r})$ with energy $E_{0}$ is obtained as the projection of the imaginary part of the self-energy $\Sigma\left(\mathbf{r}, \mathbf{r} ; E_{0}\right)$ onto the state itself (in this subsection atomic units are used, i.e., $\hbar=e^{2}=m=1$ )

$$
\Gamma_{e-e}=\tau_{e-e}^{-1}=2 \iint d \mathbf{r} d \mathbf{r}^{\prime} \psi_{0}^{*}(\mathbf{r}) \operatorname{Im} \Sigma\left(\mathbf{r}, \mathbf{r}^{\prime} ; E_{0}\right) \psi_{0}\left(\mathbf{r}^{\prime}\right)
$$

We calculate the self-energy using the $G W$ approximation $^{32}$ where only the first term in the series expansion of $\Sigma$ in terms of the screened Coulomb interaction $W$ is used. Replacing the full one-electron Green function $G$ by the noninteracting Green function we obtain the following contribution to the lifetime broadening of a $2 \mathrm{D}$ state at energy $E_{0}$ :

$$
\begin{aligned}
\Gamma_{e-e}= & 2 \sum_{E_{n \overline{\mathbf{k}}}}^{E_{0} \leqslant E_{n \overline{\mathbf{k}}} \leqslant E_{F}} \iint d \mathbf{r} d \mathbf{r}^{\prime} \psi_{0}^{*}(\mathbf{r}) \psi_{n \overline{\mathbf{k}}}(\mathbf{r}) \\
& \times \operatorname{Im} W\left(\mathbf{r}, \mathbf{r}^{\prime} ;\left|E_{0}-E_{n \overline{\mathbf{k}}}\right|\right) \psi_{0}\left(\mathbf{r}^{\prime}\right) \psi_{n \overline{\mathbf{k}}}^{*}\left(\mathbf{r}^{\prime}\right),
\end{aligned}
$$

where the sum is extended over all final states $\psi_{n \overline{\mathbf{k}}}$ with energy $E_{n \overline{\mathbf{k}}}, n$ numerates electron bands and $\overline{\mathbf{k}}$ is the 2D momentum. To avoid the very time consuming threedimensional calculations we assume that the charge density and one-electron potential varies only in the $z$ direction perpendicular to the surface and is a constant in the $(x, y)$ plane. As was shown elsewhere, ${ }^{10,25}$ this assumption works well for image states since their wave functions lie mainly in the vacuum side of the surface and electron moves, therefore, in a region with little potential variation parallel to the surface. The assumption still works reasonably well for the $s-p_{z}$ surface states on clean metal surfaces, ${ }^{14,25,33}$ because these states also do not show strong variation in the surface region. ${ }^{29,34-37}$ The $s-p_{z}$ quantum well state formed by $\mathrm{Na}$ adlayer on metal surfaces such as $\mathrm{Al}(100)$ and $\mathrm{Cu}(111)$ also shows little variation in a plane parallel to the surface. ${ }^{29,38-40}$ In our one-dimensional potential model [Eqs. (1)-(6)] the wave functions are of the form

$$
\psi_{n \overline{\mathbf{k}}}(\mathbf{r})=\frac{1}{\sqrt{A}} e^{i \overline{\mathbf{k}} \cdot \overline{\mathbf{r}}} \varphi_{n}(z)
$$

where $A$ is a surface unit cell, and the one-electron energies are

$$
E_{n \overline{\mathbf{k}}}=E_{n}+\frac{\overline{\mathbf{k}}^{2}}{2 m_{n}}
$$

Here $\varphi_{n}(z)$ and $E_{n}$ are, respectively, the normalized eigenfunctions and eigenenergies of the one-dimensional potential, which describe electron motion perpendicular to the surface. We also introduce effective masses $m_{n}$ to approximately account for the surface corrugation in a plane parallel to the surface. Assuming translational invariance of the self-energy and screened Coulomb interaction in the $(x, y)$ plane and substituting the wave functions and energies in Eqs. (10) and (11) into Eqs. (8) and (9), we obtain the following equation for the decay rate of a quasiparticle with energy $E_{0}$ and momentum $\overline{\mathbf{q}}$ in terms of the two-dimensional Fourier transforms of the self-energy,

$$
\Gamma_{e-e}=-2 \iint d z d z^{\prime} \varphi_{0}^{*}(z) \operatorname{Im} \Sigma\left(z, z^{\prime} ; \overline{\mathbf{q}} ; E_{0}\right) \varphi_{0}\left(z^{\prime}\right),
$$

where the $\operatorname{Im} \Sigma$ is

$$
\begin{aligned}
\operatorname{Im} \Sigma\left(z, z^{\prime} ; \overline{\mathbf{q}} ; E_{0}\right)= & \frac{1}{(2 \pi)^{2}} \sum_{E_{n}}^{E_{0} \leqslant E_{n} \leqslant E_{F}} \varphi_{n}^{*}\left(z^{\prime}\right) \varphi_{n}(z) \\
& \times \int \operatorname{Im} W\left(z, z^{\prime} ; \overline{\mathbf{q}}-\overline{\mathbf{k}} ; \mid E_{0}-E_{n}\right. \\
& +\Delta E \mid) d \overline{\mathbf{k}}
\end{aligned}
$$


where $\Delta E$ is

$$
\Delta E=\frac{\overline{\mathbf{q}}^{2}}{2 m_{0}}-\frac{\overline{\mathbf{k}}^{2}}{2 m_{n}} .
$$

The imaginary part of the screened interaction, Im $W$, satisfies the equation

$$
\begin{aligned}
\operatorname{Im} & W\left(z, z^{\prime} ; \overline{\mathbf{q}} ; E\right) \\
= & \iint d z_{1} d z_{2} V\left(z, z_{1} ; \overline{\mathbf{q}}\right) \operatorname{Im} \chi\left(z_{1}, z_{2} ; \overline{\mathbf{q}} ; E\right) \\
& \times V\left(z_{2}, z^{\prime} ; \overline{\mathbf{q}}\right)
\end{aligned}
$$

Here $V\left(z, z^{\prime} ; \overline{\mathbf{q}}\right)$ and $\chi(z, z ; \overline{\mathbf{q}} ; E)$ are two-dimensional transforms of the bare Coulomb interaction and the density response function of the interacting electron system, respectively. Within the random phase approximation $\chi\left(z, z^{\prime} ; \overline{\mathbf{q}} ; E\right)$ obeys the integral equation

$$
\begin{aligned}
\chi\left(z, z^{\prime}, \overline{\mathbf{q}} ; E\right)= & \chi^{0}\left(z, z^{\prime} ; \overline{\mathbf{q}} ; E\right) \\
& +\iint \chi^{0}\left(z, z_{1} ; \overline{\mathbf{q}} ; E\right) V\left(z_{1}, z_{2} ; \overline{\mathbf{q}}\right) \\
& \times \chi\left(z_{2}, z^{\prime} ; \overline{\mathbf{q}} ; E\right) d z_{1} d z_{2},
\end{aligned}
$$

where $\chi^{0}$ is the density response function for the noninteracting electron system, which can be calculated in terms of both eigenfunctions $\varphi_{n}(z)$ and eigenvalues $E_{n}$ of the onedimensional model potential. The expression we use for $\chi^{0}$ was derived by Eguiluz ${ }^{41}$ assuming free electron motion $\left(m_{n}=1\right)$ in the plane parallel to the surface. With these effective masses our calculations of $\chi^{0}$ take explicitly into account all band structure effects in the direction perpendicular to the surface, namely, the energy gap, the $s-p_{z}$ quantum well state located in this gap and image states as well. Real effective masses $\left(m_{n} \neq 1\right)$ are used in Eq. (14) to describe properly the energy and momentum transfer from the initial quasiparticle state to final states.

\section{Electron-phonon contribution}

The electron-phonon ( $e-\mathrm{ph})$ interaction will, in addition to the electron-electron $(e-e)$ interaction, reduce the lifetime of a hole created in a one-electron band. The phonon induced contribution to the lifetime broadening can be expressed ${ }^{42}$ in terms of the spectral Eliashberg functions $\alpha^{2} F(\omega){ }^{43}$ This function is the phonon density of states weighted by the $e$-ph coupling strength, taking into account phonon emission and absorption processes. We will be concerned with an initial hole created with a wave vector at the center of the surface Brillouin zone $\bar{\Gamma}$. This case corresponds to normal emission of an electron leaving a hole behind. The Eliashberg function is written for an electron state with energy $E_{0}$ as

$$
\alpha^{2} F_{0}(\omega)=\sum_{n, \nu} \int d \overline{\mathbf{q}}\left|g_{n}^{\nu \overline{\mathbf{q}}}\right|^{2} \delta\left(E_{0}-E_{n,-\overline{\mathbf{q}}} \pm \omega_{\nu \overline{\mathbf{q}}}\right) \delta\left(\omega-\omega_{\nu \overline{\mathbf{q}}}\right),
$$

where $\nu$ and $\overline{\mathbf{q}}$ denote phonon mode and phonon momentum parallel to the surface plane, respectively, $n$ is an electron band index as defined in the preceding section, and the + and - signs in the argument of the $\delta$ function correspond to phonon emission and absorption, respectively. Applying the wave functions given in Eq. (10), the $e$-ph coupling function $g$ in Eq. (17) can be written in the form

$$
\begin{aligned}
g_{n}^{\nu \overline{\mathbf{q}}}= & \sum_{\alpha} \sqrt{\frac{n_{\alpha}}{2 M \omega_{\nu \overline{\mathbf{q}}}}} \int d z \varphi_{0}(z) \vec{\epsilon}_{\nu \overline{\mathbf{q}}}\left(\mathbf{R}_{\alpha}\right) \cdot \nabla_{\mathbf{R}_{\alpha}} \\
& \times V_{e i}^{\alpha}\left(\overline{\mathbf{q}}, z-Z_{\alpha}\right) \varphi_{n}(z),
\end{aligned}
$$

where $M$ is the ion mass, $n_{\alpha}$ is the density of ions in layer labeled $\alpha, \vec{\epsilon}$ is the phonon polarization vector, $\mathbf{R}_{\alpha}=\left(\overline{\mathbf{X}}, Z_{\alpha}\right)$ is the position vector of the ion, and $V_{e i}^{\alpha}\left(\overline{\mathbf{q}}, z-Z_{\alpha}\right)$ is the parallel Fourier transform of the screened electron-ion potential. The hole decay rate, or lifetime broadening, $\Gamma$ is the sum over all scattering events which conserve energy and momentum. In the quasielastic approximation (phonon energies are small in comparison with electron energies) the Eliashberg function is the same for phonon absorption and emission. Furthermore, including the electron and phonon occupation numbers, $f$ and $n_{B}$, we obtain for the lifetime broadening 42,44

$$
\begin{aligned}
\Gamma_{e-\mathrm{ph}}= & 2 \pi \hbar \int_{0}^{\infty} \alpha^{2} F_{0}(\omega)\left[2 n_{B}(T, \omega)\right. \\
& \left.+f\left(T, E_{0}+\omega\right)-f\left(T, E_{0}-\omega\right)+1\right] d \omega .
\end{aligned}
$$

From this we easily obtain the $T=0$ result in the case when the hole binding energy exceeds the maximum phonon en$\operatorname{ergy} \hbar \omega_{\max }$,

$$
\Gamma_{e-\mathrm{ph}}=2 \pi \hbar \int_{0}^{\omega_{\max }} \alpha^{2} F_{0}(\omega) d \omega .
$$

In the literature the interest considering the electronphonon coupling is usually focused on the superconductivity of bulk materials. In this case Eq. (19) is averaged on the Fermi surface over all initial states. However, in this work we are concerned with the scattering of a specific surface hole state with a given initial energy and momentum.

An important signature of the electron-phonon interaction is the linear temperature dependence of $\Gamma_{e-p h}$ at high temperatures, which is a consequence of the $T$ dependence of the phonon occupation numbers $n_{B}(\omega) \rightarrow k_{B} T / \omega$ as $T \rightarrow \infty$. In this limit, Eq. (19) can be written

$$
\Gamma_{e-\mathrm{ph}}=2 \pi \hbar \lambda k_{B} T,
$$

where the so-called $e$-ph coupling parameter then is given by

$$
\lambda=2 \int_{0}^{\omega_{\max }} \frac{\alpha^{2} F_{0}(\omega)}{\omega} d \omega .
$$

For one monolayer of $\mathrm{Na}$ adsorbed on the surface of $\mathrm{Cu}(111)$ we have no detailed experimental or theoretical information about the phonon dispersion relations. The closest we can get, referring to experimental data, is the He scatter- 
ing experiment on the system $2-20-\mathrm{ML} \mathrm{Na}$ on $\mathrm{Cu}(100){ }^{45}$ There, flat phonon modes were found, referring to the phonon energy versus momentum parallel to the surface, as well as the substrate Rayleigh waves for thin overlayer films and the overlayer Rayleigh mode for thick adfilms. The observed flat vibrational modes are consistent with "organ pipe" type of phonon modes polarized normal to the surface.

Phonons in $1 \mathrm{ML}$ of another alkali metal, $\mathrm{Cs}$, on $\mathrm{Cu}(100)$ were recently studied in detail by Witte and Toennies. ${ }^{46}$ In addition to two overlayer modes, longitudinal mode $L$ and Rayleigh mode $R$, they also found an optical perpendicular resonance mode denoted $P$. Lattice dynamics calculations show that $L$ is strictly localized to the Cs monolayer while $R$ is less localized and $P$ even more spread into the copper substrate. However, for $1 \mathrm{ML}$ of $\mathrm{Na}$ on $\mathrm{Cu}(100),{ }^{46}$ they concluded, based on experimental data and calculations, that the $L$ mode shifts up in the bulk phonon bands, while the Rayleigh modes remain still split-off.

The phonon picture of the system 1-ML $\mathrm{Na}$ on the $\mathrm{Cu}(111)$ surface is not known. However, based on the results for $\mathrm{Cs}$ and $\mathrm{Na}$ on $\mathrm{Cu}(001)$, we have a reason to believe that surface phonon modes polarized perpendicular to the surface exist. Furthermore, we cannot exclude the possibility that these modes have a substantial weight in a few substrate layers beneath the Na layer. Information could, in principle, be obtained from $a b$ initio calculations. However, these calculations are computationally cumbersome, requiring a thick $\mathrm{Cu}$ substrate film and a large unit cell with a $3 / 2 \times 3 / 2$ adsorption structure with two different adsorption sites for the $\mathrm{Na}$ atoms, ${ }^{8,40}$ and to date have not been performed.

To estimate the $e$-ph contribution to the QWS hole lifetime broadening we therefore use Debye and Einstein models to describe the vibrational spectrum of $1 \mathrm{ML} \mathrm{Na} / \mathrm{Cu}(111)$. This estimate is based on (i) Debye and Einstein models combined with experimental $e$-ph coupling parameter $\lambda$, and (ii) an Einstein phonon model combined with a first principles pseudopotential scheme for the calculation of the deformation potential. In the first case the $e$-ph coupling function $g$ is assumed constant and thus $\alpha^{2} F$ is proportional to the phonon density of states [see Eq. (17)]. As a result the Eliashberg function and the phonon contribution to the lifetime broadening at $T=0$ [Eq. (20)] are given as follows. ${ }^{9}$

a. Bulk (3D) Debye model. For $\omega<\omega_{D}$,

$$
\begin{gathered}
\alpha^{2} F(\omega)=\lambda \omega^{2} / \omega_{D}^{2}, \\
\Gamma_{e-\mathrm{ph}}=\frac{2 \pi \hbar \lambda \omega_{D}}{3}\left(\frac{\omega}{\omega_{D}}\right)^{3} .
\end{gathered}
$$

For $\omega>\omega_{D}$,

$$
\begin{gathered}
\alpha^{2} F(\omega)=0, \\
\Gamma_{e-\mathrm{ph}}=2 \pi \hbar \lambda \omega_{D} / 3 .
\end{gathered}
$$

b. Two-dimensional (2D) Debye model that includes firstorder diagrams only. For $\omega<\omega_{D}$,

$$
\alpha^{2} F(\omega)=\frac{\lambda \omega}{2 \omega_{D}},
$$

$$
\Gamma_{e-\mathrm{ph}}=\frac{\pi \hbar \lambda \omega_{D}}{2}\left(\frac{\omega}{\omega_{D}}\right)^{2} ;
$$

for $\omega>\omega_{D} \alpha^{2} F(\omega)=0$ and

$$
\Gamma_{e-\mathrm{ph}}=\pi \hbar \lambda \omega_{D} / 2 .
$$

c. 2D Debye model that includes first- and second-order diagrams. For $\omega<\omega_{D}$,

$$
\begin{gathered}
\alpha^{2} F(\omega)=(\lambda / \pi) \omega\left(\omega_{D}^{2}-\omega^{2}\right)^{-1 / 2}, \\
\Gamma_{e-\mathrm{ph}}=2 \hbar \lambda\left(\omega_{D}-\left(\omega_{D}^{2}-\omega^{2}\right)^{1 / 2}\right) ;
\end{gathered}
$$

for $\omega>\omega_{D} \alpha^{2} F(\omega)=0$ and

$$
\Gamma_{e-\mathrm{ph}}=2 \hbar \lambda \omega_{D} .
$$

d. Einstein model.

$$
\alpha^{2} F(\omega)=\frac{\lambda \omega_{E}}{2} \delta\left(\omega-\omega_{E}\right)
$$

and

$$
\Gamma_{e-\mathrm{ph}}=\pi \hbar \lambda \omega_{E} .
$$

In Eqs. (23)-(34) $\omega_{D}$ and $\omega_{E}$ are characteristic Debye and Einstein frequencies, respectively.

The use of the Debye models described above, combined with experimentally determined $\lambda$ values, to estimate $\Gamma_{e-\mathrm{ph}}$ for holes in surface states on the (111) surface of noble metals $^{14}$ and on $\mathrm{Al}(100)$, normally leads to fairly good results. ${ }^{9}$ Assuming the conditions are similar for $1 \mathrm{ML}$ of $\mathrm{Na}$ on $\mathrm{Cu}(111)$ one can expect the same accuracy estimating $\Gamma_{e-\mathrm{ph}}$ for the QWS hole. In the following section we give the results for this estimate.

Despite successful applications of the Debye model to clean metal surfaces, ${ }^{14,48-50}$ this model does not give detailed information about the $e$-ph mechanism as it requires experimental $\lambda$ values where all the $e$-ph coupling is hidden. In more sophisticated calculations, based on Eqs. (17)-(22), the $e$-ph coupling function $g$ is computed taking into account electronic structure and real phonons (bulk and surface modes). ${ }^{9,44,47}$

To obtain some information on the $e$-ph decay mechanism we have performed a limited first-principle pseudopotential calculation, assuming a vibrational mode strictly localized to the Na layer. To calculate the $e$-ph matrix elements we adopt the electron wave functions obtained from the model potential scheme [Eqs. (1) -(6)] and an Einstein model for a transverse overlayer vibrational mode polarized normal to the surface with an energy denoted $\hbar \Omega_{0}$. Applying a total energy analysis we determined the vibrational energy $\hbar \Omega_{0}$ $=18-21 \mathrm{meV} .{ }^{8}$ For $\mathrm{Na}$ on $\mathrm{Cu}(111)$ the vibrational energy has only been measured in the coverage range $0-0.35 \mathrm{ML}$ and found to be rather constant $\sim 20 \mathrm{meV}$. ${ }^{51}$ Experimental data are available for 1-ML $\mathrm{Na}$ on $\mathrm{Cu}(100)$ and in this case the vibrational energy is $\hbar \Omega_{0}=18 \mathrm{meV} .{ }^{45}$

Our assumption of a strictly localized overlayer vibrational mode will possibly exaggerate the value of $\Gamma_{e-\mathrm{ph}}$ since 
the mode might have considerable weight on a few substrate layers. Nevertheless, in combination with the simple estimates of $\Gamma_{e-\mathrm{ph}}$ described above we are able to say something about this matter and also about the relative importance of intraband and interband scattering contributions to the phonon induced hole decay. For the QWS hole at the $\bar{\Gamma}$ point, intraband scattering obviously involves phonon emission only, while interband transitions include both phonon absorption and emission.

Important quantities for our theoretical estimate of $\Gamma_{e \text {-ph }}$ are the deformation potential $\partial V_{e i} / \partial R_{z}$ and the vibrational energy $\hbar \Omega_{0}$. We evaluate the deformation potential and the vibrational energy from a first-principles density-functional theory (DFT) calculation ${ }^{52,53}$ using the generalized gradient approximation for the exchange-correlation functional, ${ }^{54}$ applying a plane-wave basis and ultrasoft pseudopotentials ${ }^{55}$ for both $\mathrm{Na}$ and $\mathrm{Cu}^{8,40}$ The local deformation potential is calculated as

$$
\frac{\partial V_{e i}\left(R_{z}=0 ; z, \overline{\mathbf{x}}\right)}{\partial R_{z}} \approx \frac{V_{D F T}(\delta Q ; z, \overline{\mathbf{x}})-V_{D F T}(0 ; z, \overline{\mathbf{x}})}{\delta Q},
$$

where $V_{D F T}\left(R_{z}=0 ; z, \overline{\mathbf{x}}\right)$ and $V_{D F T}\left(R_{z}=\delta Q ; z, \overline{\mathbf{x}}\right)$ are the local self-consistent effective one-electron potentials when the $\mathrm{Na}$ monolayer is in the relaxed equilibrium ground state $\left(R_{z}=0\right)$ and when it has been rigidly displaced a typical vibrational amplitude $\delta Q=0.1 \AA$ in the normal direction away from the copper substrate, respectively. We have shown that this deformation potential is actually confined within a radius less than half the nearest neighbor distance in the monolayer. ${ }^{9}$ The deformation of the electron-ion potential, as the Na atoms vibrate [Eq. (35)], is taken into account as we know from detailed electron structure studies ${ }^{40}$ that there is a substantial charge transfer from the $\mathrm{Na}$ overlayer to the copper substrate. This charge transfer is responsible for the $\mathrm{Na}$ induced 2-eV decrease of the work function. In this way we include the dynamics of the screening of the ionelectron potential.

Due to the Einstein phonon mode approximation the $e$-ph coupling function in Eq. (18) simplifies considerably. The binding energies of the QWS hole are 42 and $127 \mathrm{meV}$ for the Na coverages 0.95 and $1 \mathrm{ML}$, respectively. These energies exceed the phonon frequencies and thus, for any reasonable temperature, the final states of the QWS hole will initially be occupied by an electron. The Fermi function $f$ in Eq. (19) is thus equal to 1 and the temperature dependence becomes completely determined by the phonon occupation numbers $n\left(\Omega_{0}\right)$. For the QWS hole decay we obtain

$$
\begin{aligned}
\Gamma_{e-\mathrm{ph}}= & \frac{n_{N a}}{2 M \Omega_{0}}\left\{m_{0}\left|\mathcal{M}_{00}\right|^{2}\left[1+n_{B}\left(T, \Omega_{0}\right)\right]\right. \\
& \left.+\sum_{n} m_{n}\left|\mathcal{M}_{0 n}\right|^{2}\left[1+2 n_{B}\left(T, \Omega_{0}\right)\right]\right\},
\end{aligned}
$$

where $m_{0}$ is the QWS electron band mass, $m_{n}$ is the final state band mass, $n_{\mathrm{Na}}$ is the surface density of $\mathrm{Na}$ atoms, and $M$ is the $\mathrm{Na}$ atom mass.
The $e$-ph matrix elements between initial $(n=0)$ and final (n) bands are given by

$$
\mathcal{M}_{0 n}=\int_{-\infty}^{+\infty} \varphi_{n}(z)\left\{\int \frac{\partial \widetilde{V}_{e i}}{\partial R_{z}}\left(R_{z}=0 ; z, \overline{\mathbf{x}}\right) e^{i \overline{\mathbf{k}}_{n} \cdot \overline{\mathbf{x}}} d \overline{\mathbf{x}}\right\} \varphi_{0}(z) d z,
$$

where $\widetilde{V}_{e i}$ is the electron-ion potential. In the case of a hole at $\bar{\Gamma}$ (normal electron emission), the $e$-ph coupling parameter $\lambda$ simplifies and we obtain, according to Eq. (22),

$$
\lambda=\frac{1}{2 \pi \Omega_{0}}\left[\Gamma_{0}^{\text {intra }}+2 \Gamma_{0}^{\text {inter }}\right]
$$

in terms of intraband and interband scattering at zero temperature.

\section{Calculation results}

By using the model potential of Eqs. (1)-(6) and experimental effective electron masses for the $\mathrm{QWS}^{5}$ as well as for bulk states we find $\Gamma_{e-e}^{1.0}=13 \mathrm{meV}$ for $1-\mathrm{ML} \mathrm{Na} / \mathrm{Cu}(111)$ and $\Gamma_{e-e}^{0.95}=4 \mathrm{meV}$ for $0.95-\mathrm{ML} \mathrm{Na} / \mathrm{Cu}(111)$. These values contain the correction due to the screening effect by $d$ electrons of the copper substrate evaluated following the procedure used in Ref. 14 for the clean (111) noble metal surfaces. $\Gamma_{e-e}^{1.0}$ and $\Gamma_{e-e}^{0.95}$ have been calculated at the QWS edges $-127 \mathrm{meV}$ and $-42 \mathrm{meV}$, respectively, measured with STM. $^{5}$

We evaluate $\Gamma_{e \text {-ph }}$ applying the Debye and Einstein models combined with the experimental $\lambda$ value, $\lambda=0.24$, determined by Carlsson et al. for $0.95-\mathrm{ML} \mathrm{Na} / \mathrm{Cu}(111){ }^{7}$ The value of the Debye energy, $\hbar \omega_{D}=18 \mathrm{meV}$, has been deduced from helium atom scattering measurements of $1 \mathrm{ML}$ of $\mathrm{Na}$ on $\mathrm{Cu}(100)$ (Ref. 45) and corresponds to the lower value obtained in a first-principles calculation. ${ }^{8}$ Referring to Eqs. (23)-(34) we obtain the following estimates of the phonon induced lifetime broadening. The 3D Debye model gives $\Gamma_{e-\mathrm{ph}}^{3 \mathrm{D}-\mathrm{D}}=9 \mathrm{meV}$. The 2D Debye models lead to $\Gamma_{e-\mathrm{ph}}^{2 \mathrm{D}-\mathrm{D}}$ $=7 \mathrm{meV}$ and $\Gamma_{e-\mathrm{ph}}^{2 \mathrm{D}-\mathrm{D} 2}=9 \mathrm{meV}$, respectively. Applying the Einstein model we use the Einstein energies given by the "center of gravity" of the 3D-D and 2D-D1 Debye density of states, (3/4) $\hbar \omega_{D}$ and $(2 / 3) \hbar \omega_{D}$, respectively. Thus we obtain $\Gamma_{e-\mathrm{ph}}^{3 \mathrm{D}-\mathrm{E}}=10 \mathrm{meV}$ and $\Gamma_{e-\mathrm{ph}}^{2 \mathrm{D}-\mathrm{D}}=9 \mathrm{meV}$.

The first-principles calculations described in the preceding section assuming a strictly overlayer-localized perpendicular Einstein phonon mode yields the decay rate $\Gamma_{e-\mathrm{ph}}^{D F T}$ $=22 \mathrm{meV}$ and $23 \mathrm{meV}$ corresponding to the Na coverages $0.95 \mathrm{ML}$ and $1 \mathrm{ML}$, respectively. In comparison with the estimates of the phonon induced lifetime broadening applying the Debye and Einstein models above, which gives value in the range $8.5 \pm 1.5 \mathrm{meV}$, these values are far too large. The deformation potential is localized to a limited region near the atomic positions and the QWS wave function has by far its greatest overlap with the atomic layers for the $\mathrm{Na}$ overlayer, as shown in Fig. 2. Thus, even if the phonon mode has an exponential spread to several upper substrate layers, 


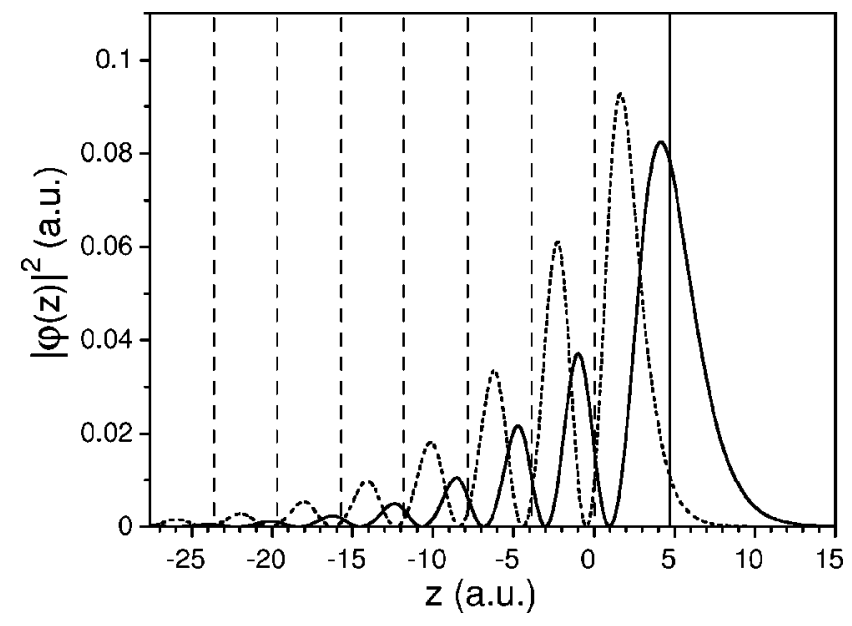

FIG. 2. Calculated probability amplitude for the QWS at 1-ML $\mathrm{Na} / \mathrm{Cu}(111)$ (thick solid line) and for the $\bar{\Gamma}$ surface state on clean $\mathrm{Cu}(111)$ (dotted line). Vertical lines indicate the atomic layer positions as in Fig. 1.

the $e$-ph matrix elements for these layers should be very small. If the amplitude of the phonon mode in the Na layer is denoted $\left|\vec{\epsilon}_{\mathrm{Na}}\right|$, we then have

$$
\Gamma_{e-\mathrm{ph}} \approx\left|\vec{\epsilon}_{\mathrm{Na}}\right|^{2} \Gamma_{e-\mathrm{ph}}^{D F T}
$$

We thus obtain $\left|\vec{\epsilon}_{\mathrm{Na}}\right|^{2} \approx 8.5 / 22.5 \approx 0.4$. From this analysis we conclude that the weight of the phonon mode is about $40 \%$ in the $\mathrm{Na}$ overlayer and $60 \%$ in the substrate. From the firstprinciples calculation we furthermore find that the contribution to the phonon induced decay rate, due to the vibrating Na layer, originates from about $70 \%$ and $30 \%$ intraband and interband electron scattering, respectively.

\section{EXPERIMENTAL PART}

\section{A. Experimental procedures}

The experiments were performed with a custom-built ultrahigh-vacuum STM operated at a temperature $T$ $=4.6 \mathrm{~K}^{56} \mathrm{Na}$ from a thoroughly outgassed commercial SAES Getters source was dosed onto a $\mathrm{Cu}(111)$ crystal cleaned by repeated sputter and annealing cycles. Although the evaporation rate was monitored with a quartz crystal microbalance we used the binding energy $E_{0}$ of the Na-induced state, which depends sensitively on the Na coverage, ${ }^{57}$ to calibrate the coverage. Following the preparation at room temperature the sample was transferred to the STM and cooled to $T=4.6 \mathrm{~K}$. Tunneling spectra of the differential conductance $d I / d V$, which resembles the local density of states, were recorded using a lock-in technique with modulation amplitudes ranging from 0.5 to $2 \mathrm{mV}_{\mathrm{pp}}$ added to the tunneling junction bias. STM was used to verify surface quality before spectroscopic measurements were performed. Spectroscopy was performed at large distances from any defects.

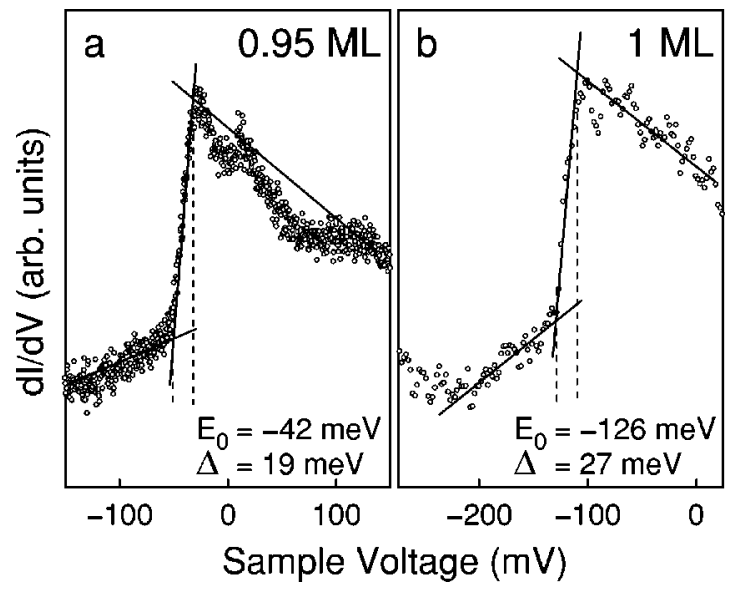

FIG. 3. $d I / d V$ spectra recorded from 0.95-ML and 1-ML Na on $\mathrm{Cu}(111)$. The bottom of the Na-induced band is clearly discernible as a sharp rise of the conductance occurring at $E_{0}=-42 \mathrm{meV}$ and $-126 \mathrm{meV}$, respectively. We define the width $\Delta$ of this rise by extrapolating the slope at the midpoint of the rise to the continuation of the conductance above and below.

\section{B. Experimental results}

In spectra of the differential conductance $d I / d V$ such as those shown in Fig. 3, the Na-induced QWS causes clear structure - a sharp rise of the conductance near $-42 \mathrm{mV}$ in the $0.95-\mathrm{ML}$ case and at $-126 \mathrm{mV}$ for $1 \mathrm{ML}$. The rapid increase reflects the onset of the contribution to the tunneling current of electrons in the quantum-well state, and, as in the case of noble metal surface states, ${ }^{13,14}$ we use the energy width of this rise to determine the inverse lifetime. To quantify the width, which we denote $\Delta$, we adopt the geometrical definition illustrated in Fig. 3, extrapolating the slope at the midpoint of the rise to the continuation of the conductance above and below the onset. As discussed in detail previously, ${ }^{13}$ when tunneling out of a two-dimensional surface electron band with parabolic dispersion the geometrical width $\Delta$ may be related to the linewidth $\Gamma$ traditionally used in photoelectron spectroscopy,

$$
\Gamma=\frac{2 \Delta}{\beta \pi} .
$$

Here, $\beta=1$ if variations in tip electronic structure and the energy and momentum dependence of tunneling matrix elements are neglected. For a more accurate estimate, we simulate the tunneling measurements and determine $d I / d V$ by numerically differentiating the current calculated within the perturbative many-body tunneling theory of Zawadowski, Appelbaum, and Brinkman, ${ }^{58}$

$$
\begin{aligned}
I(V)= & \frac{e}{\pi} \int_{S} d \mathbf{r} \int_{S} d \mathbf{r}^{\prime} \int d \omega[f(\omega, T)-f(\omega+V, T)] \\
& \times\left[(\dot{\nabla}-\vec{\nabla}) \operatorname{Im} G_{S}\left(\mathbf{r}, \mathbf{r}^{\prime} ; \omega+V\right)\right. \\
& \left.\times(\bar{\nabla}-\vec{\nabla}) \operatorname{Im} G_{T}\left(\mathbf{r}^{\prime}, \mathbf{r} ; \omega\right)\right]
\end{aligned}
$$


TABLE II. Comparison of experimental and calculated linewidths. $E_{0}$ is the experimental binding energy (all energies in $\mathrm{meV}) ; \Delta$ is the width of the surface band onset; $\beta$ is the correction factor close to unity, see text, $\Gamma_{S T M}$ is the linewidth determined according to Eq. (40) from the experimental data; and $\Gamma_{\text {calc }}$ is the calculated total linewidth adding the $\Gamma_{e-e}$ and $\Gamma_{e-\mathrm{ph}}$ contributions.

\begin{tabular}{lccccccc}
\hline \hline $\mathrm{ML}$ & $E_{0}$ & $\Delta$ & $\beta$ & $\Gamma_{S T M}$ & $\Gamma_{e-e}$ & $\Gamma_{e-\mathrm{ph}}$ & $\Gamma_{\text {calc }}$ \\
\hline 0.95 & -42 & 19 & $0.75-0.9$ & $14.5 \pm 1.5$ & 4.0 & $8.5 \pm 1.5$ & $12.5 \pm 1.5$ \\
1.0 & -127 & 27 & $0.75-0.9$ & $21.0 \pm 2.0$ & 13 & $8.5 \pm 1.5$ & $21.5 \pm 1.5$ \\
\hline \hline
\end{tabular}

where $\operatorname{Im} G_{T}$ and $\operatorname{Im} G_{S}$ are imaginary parts of Green functions for the tip and sample respectively, $f$ the Fermi occupation function, and where the surface integrals taken over the plane surface $S$ bisecting tip and sample. We use a single $W$ atom for the tip, using a large optical potential of $\sim 4 \mathrm{eV}$ to broaden the spectrum and provide a range of states into which to tunnel. For the sample we calculate $G_{S}$ using layer multiple-scattering methods (LKKR) within the atomic sphere approximation. ${ }^{59}$ Owing to the large size mismatch of $\mathrm{Na}$ and $\mathrm{Cu}$ and uncertainty in the $\mathrm{Na}-\mathrm{Cu}$ interlayer spacing (which cannot be determined "ab initio" within the LKKR approach), a large number of calculations have been performed to sample various sphere radii and structural parameters. For all these calculations we find that $\beta$ falls into the range $0.75-0.90$, including variations in $\mathrm{Na}$ coverages from $3 \times 3$ to $3 / 2 \times 3 / 2$.

Table II summarizes the results. There is a clear change of the width parameter $\Delta$ with coverage. While the experimental uncertainty of $\Delta$ is fairly small $(<2 \mathrm{meV})$, the uncertainty of the parameter $\beta$ leads to STM-based linewidths of $\Gamma_{S T M}=13-16 \mathrm{meV}$ and $19-23 \mathrm{meV}$ for $0.95-$ and $1-\mathrm{ML}$ coverage.

\section{DISCUSSION}

In Table II we compare linewidths obtained from the STM measurements with calculated ones. In view of the complexity of the processes under consideration, the agreement of the calculated total linewidths $\Gamma_{\text {calc }}$, which includes electronelectron and electron-phonon (3D-Debye model) contributions, with the measured data is reasonable.

Despite the uncertainty in converting $\Delta$ to $\Gamma_{S T M}$ there is a clear variation of the experimental linewidths. The calculations indicate that the change of the linewidth with $\mathrm{Na}$ coverage is due to a change of the $e-e$ interaction. The increase of the $e$-e contribution, moving from 0.95 to $1 \mathrm{ML}$, is due to the large increase of the QWS binding energy from 42 to 127 $\mathrm{meV}$. This will open up an increasing number of possible intraband scattering events which will increase the linewidth. Furthermore, the $e-e$ contribution to the linewidth is as important as the $e$-ph contribution which is in contrast to naive expectations based on the 3D free-electron-gas (FEG) model which leads to $\Gamma_{e-e}<1 \mathrm{meV}$ for both coverages.

In interpreting this significant difference between the FEG model and a more realistic band-structure model it is useful to recall results obtained for the surface state on clean $\mathrm{Cu}(111)$ at $\Gamma \cdot{ }^{14}$ Despite the significantly stronger binding of
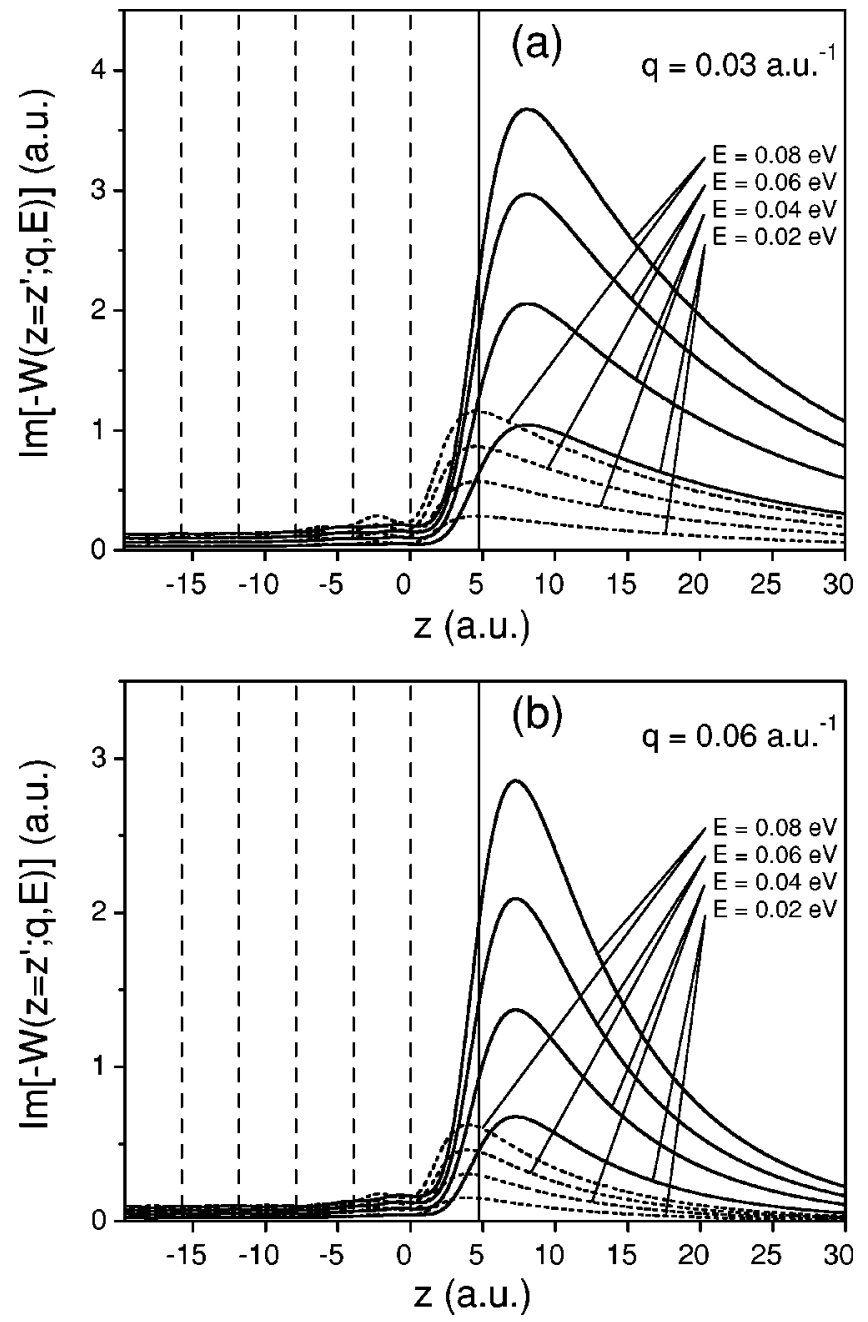

FIG. 4. Calculated imaginary part of the screened interaction, $\operatorname{Im} W\left(z, z^{\prime} ; q ; E\right)$ for $z=z^{\prime}, q=0.03$ a.u. $^{-1}$ (a), $q=0.06$ a.u. $^{-1}$ (b) and different energies for $1-\mathrm{ML} \mathrm{Na} / \mathrm{Cu}(111)$ (thick solid lines) and clean $\mathrm{Cu}(111)$ (short-dashed lines). Vertical lines indicate the atomic layer positions as in Fig. 1.

the $\mathrm{Cu}(111)$ surface state $\left(E_{\bar{\Gamma}}=-445 \mathrm{meV}\right)$ a similar value of $\Gamma_{e-e}$ was found. Again, a 3D FEG model yielded a low $\Gamma_{e-e}=2 \mathrm{meV}$ taking into account the screening of the $e-e$ interaction by $d$ electrons. This discrepancy was explained in terms of large contribution to $\Gamma_{e-e}$ from transitions within the surface state band itself, which are weighted by the enhanced imaginary part of the screened interaction that exists in the surface region. Figure 2, which displays the probability amplitude of the QWS at $1-\mathrm{ML} \mathrm{Na} / \mathrm{Cu}(111)$ and the surface state on clean $\mathrm{Cu}(111)$, highlights that the localization of the QWS to the adlayer separates the 2D state from 3D states even more efficiently than is the case of the clean noblemetal surface state.

Another factor which favors the large lifetime broadening of the QWS hole is a reduced screening of the $e$-e interaction at the surface. To illustrate the complex behavior of the screening we show in Fig. 4 the space dependence of the diagonal part of the imaginary part of the screened interaction, $\operatorname{Im} W(z, z ; \overline{\mathbf{q}} ; E)$, evaluated for $1-\mathrm{ML} \mathrm{Na} / \mathrm{Cu}(111)$ and 
for clean $\mathrm{Cu}(111)$ for different momenta and energies. One notes that, in general, $\operatorname{Im} W$ is significantly greater in the surface region than in the substrate, while in the monolayer region $\operatorname{Im} W$ is even larger than at the clean $\mathrm{Cu}(111)$ surface. Although off diagonal part of $\operatorname{Im} W\left(z, z^{\prime} ; \overline{\mathbf{q}} ; E\right)$ shows more complicated behavior than the diagonal one the conclusions drawn for $\operatorname{Im} W\left(z=z^{\prime} ; \overline{\mathbf{q}} ; E\right)$ are also valid for $\operatorname{Im} W(z$ $\left.\neq z^{\prime} ; \overline{\mathbf{q}} ; E\right)$ in the overlayer and the substrate areas.

The importance of the contribution of the $e$-ph interaction to the QWS hole decay is illustrated by the numbers in Table II. Combining estimates based on Debye and Einstein phonon models with limited first-principles calculations we find that the surface vibrational mode of $\mathrm{Na}$ on $\mathrm{Cu}(111)$ is less localized to the overlayer than previously suggested ${ }^{8}$ - according to our analysis, only about $40 \%$ of the mode is localized to the Na layer. Furthermore, the phonon induced linewidth seems to be insensitive to the variation of the Na coverage in the range 0.95 to $1 \mathrm{ML}$, the reason being that the surface phonon energies are smaller than the QWS binding energies at these coverages.

\section{CONCLUSIONS}

We have used scanning tunneling spectroscopy to evaluate lifetimes of holes in occupied quantum-well states on Na-covered $\mathrm{Cu}(111)$. Model calculations of the electronelectron and electron-phonon interactions have been used to analyze these data. We find that variations in the electronelectron scattering are responsible for the observed variations with $\mathrm{Na}$ coverage, on account of two effects. First, the large overlap of the initial and final states in the case of intraband transitions within the quantum-well state makes this decay channel particularly efficient. Second, the screened Coulomb interaction $\operatorname{Im} W$ is significantly increased in the quantumwell state. While $\operatorname{Im} W$ is already increased at the pristine $\mathrm{Cu}(111)$ surface compared to the interior of the crystal, it is yet stronger for the QWS at monolayer coverage. Combining these effects leads to comparable values of $\Gamma_{e-e}$ for the $\mathrm{Na} /$ $\mathrm{Cu}(111)$ overlayer and the clean $\mathrm{Cu}(111)$ surface state despite significantly different binding energies.

The important contribution of the electron-phonon interaction to limiting hole lifetimes is reflected by the observed large electron-phonon coupling parameter $\lambda=0.24 .^{7}$ Our combined model study and limited first-principle calculation suggest that the $e$-ph contribution is insensitive to the $\mathrm{Na}$ coverage change from 0.95 to $1 \mathrm{ML}$, whilst the weight of the active surface phonon mode in the Na layer is only about $40 \%$, in contrast to $100 \%$ suggested in earlier studies. ${ }^{8}$

\section{ACKNOWLEDGMENTS}

We acknowledge partial support by the University of the Basque Country, the Basque Hezkuntza Unibertsitate eta Ikerreta Saila, and Ministerio de Ciencia y Tecnología (Grant No. MAT 2001-0946). Financial support by the Deutsche Forschungsgemeinschaft and Deutscher Akademischer Austauschdienst is gratefully acknowledged. The work was also financially supported by the Swedish Scientific Research Council (VR). P.M.E. acknowledges support from the Max Planck Foundation; S.C. acknowledges the support of British Council.
${ }^{1}$ S.-Å. Lindgren and L. Waldén, Phys. Rev. B 38, 3060 (1988).

${ }^{2}$ T.-C. Chiang, Surf. Sci. Rep. 39, 181 (2000).

${ }^{3}$ M. Milun, P. Pervan, and D.P. Woodruff, Rep. Prog. Phys. 65, 99 (2001).

${ }^{4}$ For a review, see Th. Fauster and W. Steinmann, in Electromagnetic Waves: Recent Developments in Research, edited by P. Halevi, Photonic Probes of Surfaces Vol. 2 (Elsevier, Amsterdam, 1995).

${ }^{5}$ J. Kliewer and R. Berndt, Phys. Rev. B 65, 035412 (2001).

${ }^{6}$ N. Fischer, S. Schuppler, R. Fischer, Th. Fauster, and W. Steinmann, Phys. Rev. B 47, 4705 (1993).

${ }^{7}$ A. Carlsson, B. Hellsing, S.-Å. Lindgren, and L. Walldén, Phys. Rev. B 56, 1593 (1997).

${ }^{8}$ B. Hellsing, J. Carlsson, L. Walldén, and S.-Å. Lindgren, Phys. Rev. B 61, 2343 (2000).

${ }^{9}$ For a review, see B. Hellsing, A. Eiguren, and E.V. Chulkov, J. Phys.: Condens. Matter 14, 5959 (2002).

${ }^{10}$ For a review, see P.M. Echenique, J.M. Pitarke, E.V. Chulkov, and A. Rubio, Chem. Phys. 251, 1 (2000).

${ }^{11}$ The first monolayer of $\mathrm{Na}$ on $\mathrm{Cu}(111)$ saturates at a coverage of $4 \mathrm{Na}$ atoms per $9 \mathrm{Cu}$ atoms with a $(3 / 2 \times 3 / 2)$ unit mesh [D. Tang, D. McIlroy, X. Shi, C. Su, and D. Heskett, Surf. Sci. 255, L497 (1991); J. Kliewer and R. Berndt, ibid. 477, 250 (2001)]. We define this structure as 1 monolayer.
${ }^{12}$ R. Matzdorf, Surf. Sci. Rep. 30, 153 (1998).

${ }^{13}$ J.T. Li, W.-D. Schneider, R. Berndt, O. Bryant, and S. Crampin, Phys. Rev. Lett. 81, 4464 (1998).

${ }^{14}$ J. Kliewer, R. Berndt, E.V. Chulkov, V.M. Silkin, P.M. Echenique, and S. Crampin, Science 288, 1399 (2000).

${ }^{15}$ F. Reinert, G. Nicolay, S. Schmidt, D. Ehm, and S. Hüfner, Phys. Rev. B 63, 115415 (2001).

${ }^{16}$ J. Kliewer, R. Berndt, and S. Crampin, New J. Phys. 288, 1399 (2000).

${ }^{17}$ K.-F. Braun and K.-H. Rieder, Phys. Rev. Lett. 88, 096801 (2002).

${ }^{18}$ S. Pons, P. Mallet, L. Magaud, and J.Y. Veuillen, Europhys. Lett. 61, 375 (2003).

${ }^{19} \mathrm{Ph}$. Avouris, I.-W. Lyo, R.E. Walkup, and Y. Hasegawa, J. Vac. Sci. Technol. B 12, 1447 (1994).

${ }^{20}$ L. Bürgi, O. Jeandupeux, H. Brune, and K. Kern, Phys. Rev. Lett. 82, 4516 (1999).

${ }^{21}$ L. Vitali et al., Surf. Sci. 523, L47 (2003).

${ }^{22}$ M. Pivetta, F. Silly, F. Patthey, J. P. Pelz, and W.-D. Schneider, Phys. Rev. B 67, 193402 (2003).

${ }^{23}$ E.V. Chulkov, V.M. Silkin, and P.M. Echenique, Surf. Sci. 391, L1217 (1997).

${ }^{24}$ E.V. Chulkov, V.M. Silkin, and P.M. Echenique, Surf. Sci. 437, 330 (1999). 
${ }^{25}$ For a review, see E.V. Chulkov, V.M. Silkin, and M. Machado, Surf. Sci. 482-485, 693 (2001), and references therein.

${ }^{26}$ W. Berthold et al., Phys. Rev. Lett. 88, 056805 (2002); A. GarcíaLekue et al., ibid. 89, 096401 (2002).

${ }^{27}$ A.G. Borisov et al., Phys. Rev. Lett. 86, 488 (2001); A.G. Borisov, J.P. Gauyacq, E.V. Chulkov, V.M. Silkin, and P.M. Echenique, Phys. Rev. B 65, 235434 (2002); A.G. Borisov, A.K. Kazansky, and J.P. Gauyacq, Surf. Sci. 526, 72 (2003).

${ }^{28}$ Here we give values of the independent parameters for the model potential. The parameters $A_{1}=5.14 \mathrm{eV}, A_{10}=-9.725 \mathrm{eV}, A_{2}$ $=4.25592 \mathrm{eV}$, and $\beta=2.861933 \mathrm{a.u}^{-1}$ are valid for both 1 and $0.95-\mathrm{ML} \mathrm{Na} / \mathrm{Cu}(111)$. The other parameters are the following (a) For 1-ML Na/Cu(111): $\beta_{a d}=3.48$ a.u. $^{-1}, \quad z_{s}$ $=1.1622$ a.u. with respect to the $\mathrm{Cu}$ surface layer position, $z_{\text {ad }}$ $=-0.352$ a.u. with respect to the overlayer position. (b) For $0.95-\mathrm{ML} \mathrm{Na} / \mathrm{Cu}(111): \beta_{a d}=3.70$ a.u. $^{-1}, z_{s}=1.1626$ a.u. with respect to the $\mathrm{Cu}$ surface layer position, $z_{a d}=-0.422$ a.u. with respect to the overlayer position. Work function of $2.77 \mathrm{eV}^{6}$ and an interlayer spacing of 4.705 a.u. between the $\mathrm{Cu}$ surface layer and $\mathrm{Na}$ overlayer was used for both coverages.

${ }^{29}$ E.V. Chulkov and V.M. Silkin, Surf. Sci. 215, 385 (1989).

${ }^{30}$ P.M. Echenique and J.B. Pendry, Prog. Surf. Sci. 32, 111 (1989).

${ }^{31}$ N. Fischer, S. Schuppler, R. Fischer, Th. Fauster, and W. Steinmann, Phys. Rev. B 43, 14722 (1991).

${ }^{32}$ L. Hedin and S. Lundqvist, Solid State Phys. 23, 1 (1969).

${ }^{33}$ E.V. Chulkov, V.M. Silkin, and P.M. Echenique, Surf. Sci. 454456, 458 (2000).

${ }^{34}$ S.G. Louie, Phys. Rev. Lett. 40, 1525 (1978).

${ }^{35}$ L. Ley, G.P. Kerker, and N. Martensson, Phys. Rev. B 23, 2710 (1981)

${ }^{36}$ E.V. Chulkov, V.M. Silkin, and E.N. Shirykalov, Surf. Sci. 188, 287 (1987).

${ }^{37}$ J. Inglesfield and G.A. Benesh, Phys. Rev. B 37, 6682 (1988).

${ }^{38}$ G.A. Benesh and J.R. Hester, Surf. Sci. 194, 567 (1988).
${ }^{39}$ C. Stampfl, K. Kambe, R. Fasel, P. Aebi, and M. Scheffler, Phys. Rev. B 57, 15251 (1998).

${ }^{40}$ J.M. Carlsson and B. Hellsing, Phys. Rev. B 61, 13973 (2000).

${ }^{41}$ A.G. Eguiluz, Phys. Rev. B 31, 3303 (1985).

${ }^{42} \mathrm{G}$. Grimvall, in The Electron-Phonon Interaction in Metals, Selected Topics in Solid State Physics, edited by E. Wohlfarth (North-Holland, New York, 1981).

${ }^{43}$ G.M. Eliashberg, Zh. Éksp. Teor. Fiz. 38, 966 (1960) [Sov. Phys. JETP 11, 696 (1960)].

${ }^{44}$ A. Eiguren et al., Phys. Rev. Lett. 88, 066805 (2002).

${ }^{45}$ G. Benedek et al., Phys. Rev. Lett. 69, 2951 (1992).

${ }^{46}$ G. Witte and J.P. Toennies, Phys. Rev. B 62, R7771 (2000).

${ }^{47}$ A. Eiguren, B. Hellsing, E.V. Chulkov, and P.M. Echenique, Phys. Rev. B 67, 235423 (2003).

${ }^{48}$ B.A. McDougall, T. Balasubramanian, and E. Jensen, Phys. Rev. B 51, 13891 (1995).

${ }^{49}$ T. Balasubramanian et al., Phys. Rev. B 64, 205401 (2001).

${ }^{50}$ S.-T. Tang, Ismail, P.T. Sprunger, and E.W. Plummer, Phys. Rev. B 65, 235428 (2002).

${ }^{51}$ S.Å. Lindgren and L. Walldén, J. Electron Spectrosc. Relat. Phenom. 64/65, 483 (1993).

${ }^{52}$ P. Hohenberg and W. Kohn, Phys. Rev. B 136, 864 (1964).

${ }^{53}$ W. Kohn and L. Sham, Phys. Rev. B 140, 1133 (1965).

${ }^{54}$ J. Perdew, J.A. Chevary, S.H. Vosko, K.A. Jackson, M.R. Pedersen, D.J. Singh, and C. Fiolhais, Phys. Rev. B 46, 6671 (1992).

${ }^{55}$ D. Vanderbildt, Phys. Rev. B 41, 7892 (1990).

${ }^{56}$ J. Kliewer, Ph.D. thesis, RWTH Aachen, D-52056 Aachen, Germany, 2000.

${ }^{57}$ N. Fischer, S. Schuppler, Th. Fauster, and W. Steinmann, Surf. Sci. 314, 89 (1994).

${ }^{58}$ A. Zawadowski, Phys. Rev. 163, 341 (1967); J.A. Appelbaum and W.F. Brinkman, ibid. 186, 464 (1969).

${ }^{59}$ S. Crampin, J. Phys.: Condens. Matter 5, 4647 (1993); S. Crampin and O.R. Bryant, Phys. Rev. B 54, 17364 (1996). 